\title{
The Influence of Causal Knowledge in Two-Alternative Forced-Choice Tasks
}

\author{
Rocio Garcia-Retamero $^{1, *}$, Ulrich Hoffrage ${ }^{2}$, Stephanie M. Müller ${ }^{1}$ and Antonio Maldonado ${ }^{1}$ \\ ${ }^{1}$ Universidad de Granada, Spain \\ ${ }^{2}$ Faculty of Business and Economics, University of Lausanne, Switzerland
}

\begin{abstract}
Making decisions can be hard, but it can also be facilitated. Simple heuristics are fast and frugal but nevertheless fairly accurate decision rules that people can use to compensate for their limited computational capacity, time, and knowledge when making decisions. These heuristics are effective to the extent that they can exploit the structure of information in the environment in which they operate. They require knowledge about the predictive value of probabilistic cues. However, it is often difficult to keep track of all the available cues in the environment and how they relate to any relevant criterion. We suggest that knowledge about the causal structure of the environment helps decision makers focus on a manageable subset of cues, thus effectively reducing the potential computational complexity inherent in even relatively simple decision-making tasks. Specifically, we claim that causal knowledge can act as a meta-cue for identifying highly valid cues and help to estimate cue-validities. Causal knowledge, however, can also bias people's decisions. We review experimental evidence that tested these hypotheses.
\end{abstract}

Keywords: Causal Knowledge, Information Search, Belief Revision Model, Take the Best, Decision Making, Judgment.

\section{INTRODUCTION}

When people are faced with a decision, it is often impossible to consider all of the available alternatives and to gather and process all of the information about those alternatives. For instance, to buy a laptop, most people would not consider every model that exists on the market, but winnow down the set of options to inspect closer using features such as price and quality. They might not analyze all features of the remaining laptops either, but request only certain cues to decide which one to buy $[1,2]$. Such decisions are fast because they do not involve much computation, and they are frugal because they only search for some of the available information in the environment [3].

Previous research has shown that people-in particular in situations in which they are not able to process all available information in the environment $[4,5]$ —often use mental models about cause-effect relations when determining which cues to consider [6-10]. Consumers, for instance, often believe that high product quality is associated with high production costs, resulting in higher prices. Thus, a customer may believe that the price-level predicts the quality, exclusiveness, or abstract value of a purchased object due to its production expenses [11]. In this paper, we posit that such knowledge about the causal structure of the environment can help people to reach satisfying decisions. Specifically, we analyze the impact of causal knowledge in two-alternative forced choice tasks and

*Address correspondence to this author at the Facultad de Psicologia, Universidad de Granada, Campus Universitario de Cartuja s/n, 18071 Granada, Spain; Tel: +34 958 246240, Fax: +34 958 246239;

E-mail: rretamer@ugr.es present, after a theoretical introduction, various findings and insights that are relevant to this topic.

In general, the decision making literature that focuses on the influence of causal beliefs suggests that such beliefs are like a double-edged sword: They can help or hinder. Some authors [11-13] conclude that prior beliefs boost peoples' covariation assessment and may increase decision accuracy if the causal beliefs are used as hypotheses that are tested on data [7, 12, 14-17]. Specifically, assessments of relationships between events that are guided by causal beliefs, such as the relationship between price and quality, are more accurate than belief-free judgments about abstract stimuli, especially when the data are noisy $[12,13]$. These findings suggest that causal beliefs can have beneficial effects.

Other findings, however, suggest that such beliefs can also have detrimental effects. For instance, it seems that objective correlations can only be assessed correctly when relevant prior beliefs are absent or congruent with the empirical evidence [18-20]. Moreover, identical objective correlations can be judged very differently depending on whether prior knowledge about the relationship between a cause and an effect conflicts with empirical evidence or not. For instance, participants in a study by Evans, Clibbens, Cattani, Harris, and Dennis [21, 22] were provided with information compatible, incompatible, or neutral with their prior beliefs. The results showed that their beliefs only improved judgments when the empirical evidence was compatible. An explanation for this result may be that participants overvalued prior beliefs when assessing actual contingencies [23-25]. In that way, only information confirming their prior beliefs 
was taken into account, whereas conflicting information was ignored.

Various theoretical approaches have been used to shed more light on the relation between causal beliefs and covariation information [for overviews, see 26-29]. Two approaches are particularly worth mentioning. The first conceptualizes a causal relationship as a function of the associative weights $[30,31]$ or the statistical relationship [32] between cues and outcomes acquired during previous training. This approach implies a bottom-up learning process. In contrast, the second approach presumes an abstract knowledge of causality, which allows individuals to assess a relation when presented with covariation data $[33,34]$.

There are also several theoretical attempts that integrate these two approaches - for instance, the belief revision model (BRM) [35, 36, see 24, 37 for other attempts]. In this model, previous knowledge about causation is not an absolute filter of the new covariation data. Instead, it represents an anchor adjusting the beliefs or classifying new evidence, similar to an earlier attempt on belief updating by Hogarth and Einhorn [38].

Finally, another approach addressing causal relations are causal Bayesian networks [39-41]. To apply such networks sufficient information about the environmental structures needs to be provided. These networks are displayed through directed acyclic graphs in which the nodes represent the variables (types of events or states of the world) and the edges (arrows) represent the direct causal relations or probabilistic dependence between those variables (see also [10]). A problem with causal Bayesian networks is computational intractability: When fed with large scale data sets, including thousands of variables, it is essentially impossible for these networks to identify the causal structure underlying the data.

\section{THE FAST AND FRUGAL HEURISTICS APPROACH AND THE PROBLEM OF CUE SELECTION}

A prominent approach in decision making is the fast and frugal heuristics research program proposed by Gigerenzer and the ABC Research Group [3, 42-46].

One of the fast and frugal heuristics is take-the-best [45, 46]. This heuristic is designed for two-alternative forcedchoice tasks and can be used to infer which of two alternatives has a higher value on a quantitative criterion, such as which of two university professors earns more money. The alternatives are described on several dichotomous cues such as gender or whether the professor is on the faculty of a state or a private university. These cues allow making probabilistic inferences about the criterion. Similar to other fast and frugal heuristics of this research program, take-the-best is constructed from building blocks (i.e., precise steps of information gathering and processing involved in making a decision). Specifically, this heuristic has a search rule, which defines the order of information search (take-the-best looks up cues in the order of their validity, i.e., the probability that a cue will point to the correct decision given that it discriminates between the alternatives); a stopping rule, which specifies when to stop the search (take-the-best stops after the first discriminating cue); and a decision rule, which specifies how to use the gathered information when it comes to making a decision (take-the-best chooses the alternative favored by the first discriminating cue).

The take-the-best heuristic has been subjected to empirical tests in a number of studies [47-54]. There is accumulating experimental evidence for the use of this heuristic, especially under high information acquisition costs $[50,55]$, time pressure $[54,56]$, and when participants have previous knowledge and experience in the domain [57, 58]. Newell, Weston, and Shanks [59] tested to what extent participants' behavior was consistent with take-the-best's building blocks. Their results revealed that only $75 \%$ of participants followed take-the-best's search rule (cues hierarchy established by validity) and its stopping and decision rules were obeyed in $80 \%$ and $89 \%$ of the trials, respectively [see also 53].

However, these experimental results on the use of takethe-best need to be qualified [see also 60]. In many of these studies, participants were encouraged to use cues in the order of their validity by being informed about cue validities or the validity order $[48,49,59,61]$. When search by validity was tested against alternative search orders, validity was not the search criterion that predicted participants' searches best [52]. Instead, it seemed to be the case that participants used simple rules for ordering cues based on trial-by-trial learning $[62,63]$. The cue orderings established through such rules do not necessarily converge toward the cue ordering established by validity. Participants, therefore, might have had difficulties computing cue validities and then searching for cues accordingly, even though relatively few cues (i.e., four to six) were available in those experiments.

The problem of searching for good cues seems to be even more severe when one considers that in most situations there are myriad potential cues that could be used to make a decision, and it is practically impossible to keep track of them all and to compute their validities for any potentially relevant criterion [64]. Cue selection is further complicated if potential combinations of cues (i.e., compound cues) are taken into account [47]. Yet sometimes an accurate decision requires people to do so [65]. For example, some medications might have side effects, such as nausea, if ingested together with alcohol, whereas neither the drug nor the alcohol would cause any problems if ingested alone (of course, this would also depend on the amount of alcohol or drugs that is consumed). As a consequence, a strategy that processes all possible cues would be computationally too demanding. It is also not plausible to assume that the brain comes "prewired" to represent each of the possible cues to predict a criterion.

In line with other authors $[7,10,60]$, we hypothesize that people do not process all possible cues in their natural environments but rather use their causal knowledge-i.e., their knowledge about causal relationships between events in the environment - to focus on a small and manageable subset of relevant cues. We further expect that causal knowledge might also aid learning of cue validities. In sum, causal knowledge might allow decision makers to deal adaptively with the huge number of cues that appear in the environment and to select only those that are potentially relevant. In the remainder of this paper, we offer more precise predictions about how causal knowledge can influence decision-making processes and review experimental tests of these predictions. 


\section{THE ADAPTIVE VALUE OF KNOWLEDGE ABOUT THE CAUSAL TEXTURE OF THE ENVIRONMENT}

When it comes to decision-making, we hypothesize that causal knowledge is advantageous for two reasons. First, causal knowledge might act as a meta-cue that enables people to identify or to determine valid cues in the environment. Second, causal knowledge might help to specifically focus on certain cue-criterion correlations, which, in turn, facilitates learning of cue validities. In the following, we elaborate on these advantages in more detail.

Considering the first advantage, we estimate that cues that are causally linked to the criterion tend to be more valid than cues lacking such a connection to the criterion [7, $10,33,66,67]$. For instance, lung cancer (here, an effect) is more likely to be predicted from a well-established smoking habit (i.e., a cause) than from yellowed fingers (i.e., a second effect of the common cause; see [68]). Furthermore, correlations between events that are causally linked are likely to be more robust across environments (i.e., less sensitive to contextual changes) than those without such a connection $[69,70]$. Following our example, the correlation between smoking and lung cancer would be more robust across different series of patients than the correlation between lung cancer and yellowed fingers would be. We could expect this to be the case even if we control for other alternative causes that could bring about yellowed fingers (e.g., being a painter) that might reduce their predictability for lung cancer. We hypothesize that this asymmetry between causal and non-causal cues that holds in the physical world would be reflected in human cognitive processes. We therefore expect decision makers to use their causal knowledge as a meta-cue for selecting highly valid and robust cues in the environment.

Secondly, causal knowledge might reduce the number of cue-criterion correlations to keep track of when computing cue validities [55]. This hypothesis is supported by research using multiple cue probability learning. In this paradigm, participants have to predict the criterion of a given object from multiple cues that are probabilistically related to this criterion. Previous empirical studies that use this paradigm (see [71] for a review) suggest that cues interfere with each other when participants try to learn their validities concurrently. For instance, the presentation of irrelevant cues in such a task reduces the utilization of valid cues and, consequently, the accuracy of people's judgments [72, 73]. An explanation for this finding, which can be observed even after a large number of learning trials, suggests that the irrelevant cues made it harder for participants to identify and focus on the valid cues. In contrast, when participants have the opportunity to learn cue-criterion relationships sequentially (i.e., for one cue after another), their judgments correspond more closely to the ecological correlations [74]. Based on these results, we suggest that in multiple-cue settings people with access to causal knowledge might be able to focus on certain (causal) cues, which in turn might facilitate cue validity learning.

Note, however, that causal knowledge about the cues in the environment also has to be learned [10]. Our argument, therefore, only holds if the acquisition of causal knowledge is simpler than cue validity learning. We think that this is in fact the case. Consider, for instance, learning of causal Bayesian nets. Such learning is certainly not necessarily simple, but it could be simplified if prior specific or abstract domain knowledge about the structure of the environment (e.g., causal directionality) constrains the number of potential causal relations that need to be considered $[40,75,76] .{ }^{1}$

Similarly to other scholars $[7,16,17]$, we hypothesize that causal knowledge might allow decision makers to constrain the countless number of cues that appear in a particular environment to a subset of cues that are more likely to have a high predictive value. In the following sections, we review some experiments that tested whether causal knowledge helps people to select a subset of reliable cues and whether it aids learning of cue validities.

\section{CAUSAL KNOWLEDGE AS AN AID IN CUE SELEC- TION}

Recent findings on causal knowledge in decision making stress the difference between observations and interventions [7, 10, 77]. Garcia-Retamero, Wallin, and Dieckmann [66] offer another attempt to examine the impact of causal information about cue-criterion relationships on decision-making processes. Specifically, these authors analyzed whether causal knowledge about the cues in the environment had an effect on the selection of a subset of cues that were used to make decisions and whether it facilitates the computation of cue validities.

Based on the assumption that causal knowledge helps to identify highly valid cues in the environment, GarciaRetamero, Wallin, and Dieckmann [66] hypothesized that participants would look up cues that were causally connected to the criterion (in short, causal cues) earlier than non-causal cues, even when these cues had the same validity. Participants were also expected to rely on causal cues to a greater extent than on non-causal cues in their decisions, and to be more confident and faster in their decisions when causal cues were available than when no causal cues were available. On the other hand, given that causal knowledge reduces the number of cue-criterion relationships to keep track of to compute validity, those authors hypothesized that participants would be more exact in their validity estimates for causal than for non-causal cues and, consequently, would also be more accurate in their inferences.

Two experiments test these hypotheses: The first tested the prediction that causal cues are preferred over non-causal cues, the second tested whether this was still the case if participants were allowed to learn cue validities after having been informed which cues were causally linked to the criterion. The experiments were computer-based and used twoalternative forced-choice tasks (see Fig. 1). On each trial, participants were presented with two alternatives (i.e., two species of insects) and had to decide which would show a higher criterion value (i.e., which would do more damage to a crop). To make this decision, they could look up information on up to four cues (i.e., properties of the insects, such as

\footnotetext{
${ }^{1}$ Along these lines, research in the field of artificial intelligence has recently proposed a number of algorithms capable of easily inferring causal relations from covariation patterns (e.g., the TETRAD II program; $[89,90])$. These algorithms use causal models to generate a certain pattern of statistical dependencies and then search for certain clues that reveal fragments of the underlying structure. These fragments are pieced together to form a coherent causal model.
} 


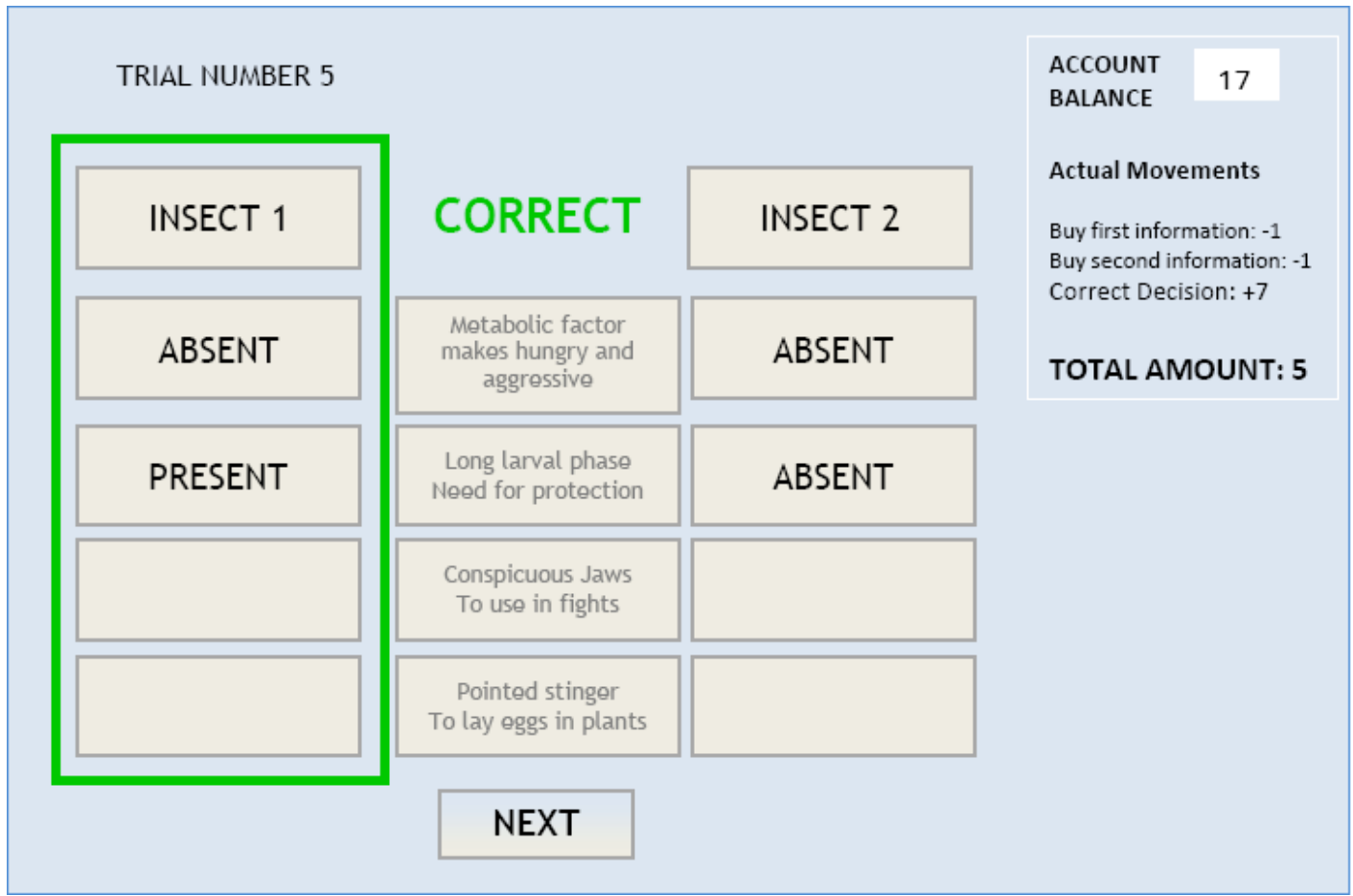

Fig. (1). Screenshot of the experimental interface (translated from German). On this trial, the participant began by accessing whether the insects had a specific metabolic factor. This cue did not discriminate between the two insects-none of them showed the metabolic factor. The participant then accessed whether the insects had a long larval phase. This cue showed a positive value for insect 1 and a negative value for insect 2 . The participant responded that insect 1 was more likely to do greater crop damage, which was a correct response. The participant earned 5 points $(7-1-1)$ in total on this trial.

the presence or absence of a particular metabolic factor), represented by small boxes on the screen that could be clicked to retrieve information (see also [48, 49, 55, 56], for similar experimental procedures).

Two of these cues had a high validity (.85) and the other two had a low validity (.65; see Table 1). Whether a specific cue had a high or a low validity was counterbalanced across participants. All four cues had a discrimination rate of $.56{ }^{2}$ Causal knowledge was manipulated between-subjects. In the causal group, participants were told that two of the cues were causally realted to the criterion (e.g., "the metabolic factor makes the insects hungry and aggressive"). These formulations suggested an underlying causal mechanism that went beyond the possible covariation between the cue and the criterion. The remaining two cues were neutral and participants were informed that they were not causally linked to the criterion (e.g., "the metabolic factor leads to green and blue coloration of the insects' body"). Which cues were causally linked to the criterion and which were neutral was counterbalanced across participants. Moreover, the two experimental factors, cue validity and causal knowledge, were completely crossed within participants so that for each participant, one of the causal cues had a high validity and the other had a low validity, and one of the neutral cues had a high validity and the second one had a low validity (Table 1). In the control group, information about all four cues was neutral. A pretest confirmed that the causal cues, but not the

${ }^{2}$ The discrimination rate of a cue is the proportion of paired comparisons in which the two decision alternatives have different value for that cue [45]. neutral cues, were indeed perceived as having a strong causal effect on the criterion.

In the first experiment, participants went through a decision phase in which the absence or presence of the cues (for each insect) was not automatically displayed, instead they had to actively access information for one cue after another. When a cue was accessed (at the cost of 1 Eurocent) the cue values (presence/absence) of both alternatives (insects) were shown. After having accessed at least one cue, participants were allowed to stop their cue search and decide for one of the alternatives (insects). Subsequently, feedback was provided whether their decision was correct (if so, they earned 7 Eurocents). At the end of the experiment, participants estimated the validity of each cue. In the second experiment, participants entered the decision phase only after they had gone through a learning phase in which the values of the four cues were provided automatically and in which participants could learn the validities of these cues.

In line with the authors' hypothesis, participants in Experiment 1 preferred to start searching for causal cues, regardless of the cue validity. Altogether, that is, across all the cues they accessed, they also favored the causal cues more often than the neutral cues. Moreover, they were faster and more confident in their decisions when they could rely on causal cues as compared to trials in which only neutral cues discriminated. Finally, participants were better in estimating the validities of the causal cues than of the neutral cues. Note that participants showed a preference for causal over neutral cues although they could learn via feedback which cues were 
Table 1. Design of Experiments 1 and 2

\begin{tabular}{|c|c|c|c|}
\hline \multirow{2}{*}{} & \multicolumn{2}{|c|}{ Experimental Group } & Control Group \\
\cline { 2 - 4 } & \multicolumn{2}{|c|}{ Information about the cue-criterion relation } \\
\hline \hline High Cue validity & Causal & Neutral & Neutral \\
\hline Low Cue validity & Cue 1 & Cue 2 & Cue 1 , Cue 2 \\
\hline
\end{tabular}

Note. In the experimental group, cue validity and information about the cue-criterion relation (causal knowledge) was manipulated within-participants. Which cue was assigned to which of the resulting four conditions was counterbalanced across participants. In the control group, no causal information was given, only cue validity was manipulated

reliable predictors (i.e., had high validity) of the criterion throughout the decision-making phase.

When participants in Experiment 2 had the opportunity to learn about cue validities before the actual decision-making phase, their search processes were influenced by both causal information and validity. More precisely, participants who had access to causal information (the causal group) preferred to search for the causal high-validity cue over the rest of the cues. Furthermore, these participants became more accurate in their decisions and were also more accurate, across all cues, when estimating cue validities. Overall, the higher frugality and accuracy in the causal group led to a higher final payoff than in the control group.

In sum, the experimental results suggest that participants may use information about which cues are causally related with the criterion to zoom in on a manageable subset of cues and to learn their validities more accurately.

\section{THE FLEXIBILITY OF CAUSAL BELIEFS: WHEN PREVIOUS BELIEFS CONFLICT WITH EMPIRICAL EVIDENCE}

Based on these results, Garcia-Retamero, Müller, Catena, and Maldonado [78] went one step further and investigated whether the relative impact of causal beliefs and empirical evidence on decision making can be altered by previous experience. Two experiments were set up as a series of twoalternative forced-choice tasks, framed as medical diagnostic tasks. In each trial, participants were asked to decide which of two patients would show a higher degree of allergic dermatitis. To make each decision, four cues were available that described both patients and participants had to search for this information.

The design and the procedure were similar to the experiments mentioned above: To analyze the influence of causal beliefs, participants were instructed that two of the four presented cues were causally linked to the criterion ("causal cues"). Instructions for the remaining two cues did not provide any causal link to the criterion ("neutral cues"). For instance, a cue containing the information that the patients ingested a certain prescription drug (Rifastan pills) could either be causal ("an antibiotic, which could lead to skin swelling") or neutral ("vitamin C tablets, which are crucial for sight"). A pretest confirmed that causal-but not neutral-cues were perceived to have a strong causal power.
The impact of the empirical evidence was examined by manipulating cue validities within-subjects: Two of the four available cues (one causal and one neutral cue) had high validity (i.e., 0.9 in both experiments); the remaining two cues had low validity (i.e., 0.6 in Experiment 1 and 0.1 in Experiment 2; see also Table 2). All four cues had a discrimination rate of $.59^{2}$ and inter-cue correlations were close to zero.

At the beginning of the experiment, some of the participants underwent pre-training with either causal (pre-causal group) or neutral cues (pre-neutral group; see also Table 2). During the pre-training, the cue values for each patient were displayed automatically —no cue search was required. Both groups were asked to make 60 decisions and outcome feedback was provided. Members of the causal control group did not receive any pre-training. Thereafter, both groups of participants completed a decision phase similar to that described above.

The results of the experiments by Garcia-Retamero, Müller, Catena, and Maldonado [78] revealed that the impact of causal beliefs and empirical evidence depends on both the experienced pre-training and the cue validity. While participants without any pre-training relied mainly on their causal beliefs-favoring causal over neutral cues-pre-training with causal cues led to a clear preference for the causal highvalidity cues. Increasing the difference between the validities of the cues reduced the influence of the causal beliefs in both groups: This manipulation led first to decisions in favor of the causal high-validity cue, and secondly to decisions in favor of the neutral high-validity cue. Finally, when participants received pre-training with neutral cues (i.e., not causally linked to the criterion), their decisions were primarily based on the high-validity cues, regardless of their induced causal or neutral relation to the outcome. These results could be observed in both experiments and suggest-in line with other research $[10,16,17,77]$ - that it is necessary to consider the joint effects of causal beliefs and empirical evidence to explain the flexibility involved in human inferences.

We can conclude from these findings that participants rely on their causal beliefs by default - especially when the validities of the cues that are supposed to be causally related to a criterion are high. In this case, participants did not take the cue validities of neutral cues into account. However, when participants received pre-training with neutral cues (i.e., not causally linked to the criterion), they became more 
Table 2. Design of Experiments1 and 2

\begin{tabular}{|c|c|c|c|c|}
\hline Experimental Groups & Instructions & Cues Pre-Training & Instructions & Cues Decision Task \\
\hline Control Causal Group & & --- & Causal & $\begin{array}{l}\text { Causal high validity } \\
\text { Causal low validity } \\
\text { Neutral high validity } \\
\text { Neutral low validity }\end{array}$ \\
\hline Pre-Causal Group & Causal & $\begin{array}{l}\text { Causal high validity } \\
\text { Causal low validity }\end{array}$ & Causal & $\begin{array}{l}\text { Causal high validity } \\
\text { Causal low validity } \\
\text { Neutral high validity } \\
\text { Neutral low validity }\end{array}$ \\
\hline Pre-Neutral Group & Neutral & $\begin{array}{l}\text { Neutral high validity } \\
\text { Neutral low validity }\end{array}$ & Causal & $\begin{array}{l}\text { Causal high validity } \\
\text { Causal low validity } \\
\text { Neutral high validity } \\
\text { Neutral low validity }\end{array}$ \\
\hline
\end{tabular}

sensitive to the validity information (i.e., they were able to discriminate high-validity from low-validity cues) and additional information about causal mechanisms failed to have further relevance. The neutral pre-training could have evoked participants' preference for the cue validities independent of causal information. Interestingly, when high validity cues differed substantially from low validity cues (up to the point where some of the cues were almost not related to the criterion), decisions were mainly based on the highvalidity cues, especially the cue that was causally linked to the criterion. Taken together, for participants who received pre-training with neutral cues or cues that provided conflicting information with previous causal beliefs, responses were mainly influenced by cue validities and-to a lesser extent - by causal beliefs.

\section{GENERAL CONCLUSIONS}

The reviewed research confirms what we stated in the introduction: causal knowledge about the causal structure of the environment is like a double-edged sword-it can help or hinder. Causal knowledge helped people to focus on a small and manageable subset of cues. It strongly influenced which cues were looked up, in which order they were looked up, and which of them were used to make decisions. Causal knowledge also facilitated cue validity learning - not an easy task, as Juslin and Persson [64] pointed out. Taken together, these findings suggest that causal knowledge can effectively reduce the computational complexity inherent in decisionmaking tasks. At the same time, it should be pointed out that participants who were equipped with causal knowledge and who did not have an opportunity to learn the cue's validities before making decisions preferred causal, low-validity cues over neutral, high-validity cues, even though they received feedback after each decision.

Seen through the lens of the fast and frugal heuristics framework, causal knowledge helps people to select valid cues in the environment, which might be placed in a high position in the cue ordering, that is, in the hierarchy of cues that is accessed by the search process of a decision-making strategy (see also [60]). To the extent that the feedback about whether a decision was correct or incorrect leads to an updating of cue validities, the cue ordering might consequently be updated as well. In this sense, causal beliefs can be perceived as hypotheses to be tested and updated with empirical data (see also [79, 80]). Consequently, causal beliefs might act as hypotheses that constrain cue selection to make decisions - whether these beliefs are confirmed or disconfirmed depends on the experience with the selected cues in the environment. In line with this result, Fugelsang, Stein, Green, and Dunbar [81] showed that even scientists are not immune against overvaluing their initial beliefs when testing their hypotheses on new data. Their results reveal that only great amounts of disconfirming evidence have the power to affect the original theory proposed by researchers.

Are our conclusions about the beneficial effect of causal knowledge restricted to the family of fast and frugal heuristics? Our intuition is that the present approach might also be extended to other decision strategies. Causal knowledge possibly could also help to reduce the computational complexity inherent in more demanding strategies for making decisions such as the weighted additive model (WADD) - a compensatory strategy that uses cue validities as weights [82]. However, contrary to fast and frugal heuristics, WADD and other compensatory strategies do not model the search process. That is, they strictly assume that all the relevant and necessary information to make decisions is available to the decision maker. Yet, as we mentioned above, this is, in fact, often not the case and thus people would have to actively search for information. We find it difficult to see how people using such compensatory strategies could use their causal knowledge to select from the wide range of candidate cues in the environment those that are highly valid. If cue search and selection is no longer driven by the strategy that is used, how would causal knowledge aid learning of cue validities? Briefly, simplification is not an inherent feature of these decision models. Consequently, in their present form, they could not benefit from the advantages of causal knowledge 
we pointed out above. The belief revision model [35, 83], for instance, tries to integrate prior beliefs with empirical evidence: A prior belief serves as an equivalent to causal knowledge, whereas new empirical evidence stands for the presented covariation data. Increasing the initial prior belief/causal knowledge and decreasing the reliability of the empirical evidence/covariation data can explain the strong impact of previous beliefs on causal decisions via simulation (see also [78]). The presence of causal knowledge is vital as it directs the search for information, facilitates the learning of cue validities, and improves decision accuracy. Not providing such knowledge in an experiment will make decision makers appear less competent than they would be in their natural environment in which such information is frequently available.

In fact, causal knowledge has a large impact on peoples' daily decisions and behavior. Consider stereotypes, for example. Stereotypes represent commonly shared causal knowledge about a certain social group that indicate their attributes, roles, and behaviors [84]. Once a stereotypic belief is implemented in someone's perception of the world, it is highly persistent to contradicting information or to breaking the "stereotypic habit" [85]. People stick to their initial beliefs for quite some time even if these are not supported by the environment. Extended practice in non-stereotypic responding, however, can lead to a decrease in the activation of stereotypes [85] — which is similar to the pre-training in one of our studies.

Another example comes from marketing strategies: living in a consumer society, most people are overwhelmed by the amount of certain products offered (e.g., laptop computers). People might therefore search only for specific qualities of a product, and in this case advertisement starts to play a significant role in "facilitating" peoples' decision making processes [86]. Advertisements aim to provide customers with causal knowledge connecting a cue with a criterion (e.g., a brand with quality) and "help" them to find the right product out of the confusing market. Adopting a more general perspective, it becomes obvious that not only companies but also political parties or other organizations try to provide the public with causal information to influence decision making [87]. For instance, even though the power of propaganda has often been underestimated, it is frequently used as a tool for social control and political indoctrination [88]. Our research does not suggest that consumers and citizens should suppress their causal knowledge and become naïve scientists examining all empirical data in the environment. First, in light of the advantages of causal knowledge this would not be desirable, and second, in light of the empirical evidence reviewed above it would be naïve to believe that this was possible in the first place. However, people could benefit from being aware of the strong impact of their causal knowledge on decisions and scrutinize their initial beliefs more often-especially when judging others or making important life decisions.

In general, it should now be clear that decisions are not only based on what can be learned, following a bottomup approach, by inspecting the empirical evidence in the environment. Rather, decisions are also influenced, in a top-down fashion, by causal knowledge. Therefore, any approach that tries to explain decision making should incorporate peoples' capacity to learn about causal structures.

\section{AUTHOR NOTE}

MICINN grants to R. Garcia Retamero (Ref: PSI200802019) and to A. Maldonado (Ref: PSI2009-12217, SEJ200611906).

\section{ACKNOWLEDGEMENTS}

We would like to thank the reviewers for their excellent comments on a previous version of this manuscript and Chris White for his careful editing.

\section{REFERENCES}

[1] Fasolo B, McClelland GH, Todd PM. Escaping the tyranny of choice: When fewer attributes make choice easier. Market Theory 2007; 7: 13-26.

[2] Reisen N, Hoffrage U, Mast F. Identifying decision strategies in a consumer choice situation. Judgm Decis Mak 2008; 38: 64158.

[3] Gigerenzer G. Rationality for mortals. New York: Oxford University Press 2008 .

[4] Kahneman D, Slovic P, Tversky A. Judgment under uncertainty: Heuristics and biases. Cambridge UK: Cambridge, University Press 1982.

[5] Simon HA. Invariants of human behavior. Annu Rev Psychol 1990; 41: 1-19.

[6] Kahneman D, Tversky A. Prospect theory: an analysis of decision under risk. Econometrica 1979; 47: 263-91.

[7] Sloman SA, Hagmayer Y. The causal psycho-logic of choice. Trends Cogn Sci 2006; 10: 407-12.

[8] Tversky A, Kahneman D. Judgment under uncertainty: Heuristics and biases. Science 1974; 185: 1124-31.

[9] Tversky A, Kahneman D. The framing of decisions and the psychology of choice. Science 1981; 211: 453-8.

[10] Waldmann MR, Hagmayer Y, Blaisdell AP. Beyond the information given: Causal models in learning and reasoning. Curr Dir Psychol Sci 2006; 15: 307-11.

[11] Alba JW, Broniarczyk SM, Shimp TA, Urbany JE. The influence of prior beliefs frequency cues and magnitude cues on consumers' perceptions of comparative price data. J Consum Res 1994; 21: 219-35.

[12] Baumgartner H. On the utility of consumers' theories in judgments of covariation. J Consum Res 1995; 21: 634-43.

[13] Wright JC, Murphy GL. The utility of theories in intuitive statistics: the robustness of theory-based judgments. J Exp Psychol Gen 1984; 113: 301-22.

[14] Garcia-Retamero R. The influence of knowledge about causal mechanisms on compound processing. Psychol Rec 2007; 57: 295306.

[15] Garcia-Retamero R, Hoffrage U. Influencia del conocimiento causal en los procesos de toma de decisiones. Rev Mex Psicol 2009; 26: 103-11.

[16] Meder B, Hagmayer Y, Waldmann MR. Inferring interventional predictions from observational learning data. Psychon B Rev 2008; 15: 75-80.

[17] Meder B, Hagmayer Y, Waldmann MR. The role of learning data in causalreasoning about observations and interventions. Mem Cogn 2009; 37: 249-64.

[18] Billman D, Bornstein BH, Richards J. Effects of expectancy on assessing covariation in data: "Prior belief" versus "meaning". Organ Behav Hum Decis Process 1992; 53 74-88.

[19] Nisbett RE, Ross L. Human inference: Strategies and shortcomings of social judgment. Englewood Cliffs NJ: Prentice-Hall 1980.

[20] Alloy LB, Tabachnik N. Assessment of covariation by humans and animals: The joint influence of prior expectations and current situational information. Psychol Rev 1984; 91: 112-49.

[21] Evans JStBT, Clibbens J, Cattani A, Harris A, Dennis I. Explicit and implicit processes in multicue judgment. Mem Cogn 2003; 31: 608-18.

[22] Evans JStBT, Clibbens J, Harris A. Prior belief and polarity in multicue learning. Q J Exp Psychol 2005; 58A: 651-65. 
[23] Chapman LJ, Chapman JP. Genesis of popular but erroneous psychodiagnostic observations. J Abnorm Psychol 1967; 72: 193204.

[24] Fugelsang JA, Thompson VA. A dual-process model of belief and evidence interactions in causal reasoning. Mem Cogn 2003; 31: 800-15.

[25] Klayman J. Varieties of confirmation bias. Psychol Learn Motiv 1995; 32: 385-417.

[26] Ahn W, Kalish CW. The role of mechanism beliefs in causal reasoning. In: Keil FC, Wilson RA, Eds. Explanation and cognition, Cambridge MA: MIT Press 2000; pp. 199-225.

[27] De Houwer J, Beckers T. A review of recent developments in research and theories on human contingency learning. Q J Exp Psychol 2002; 55B: 289-310.

[28] Perales JC, Catena A. Human causal induction: a glimpse at the whole picture. Eur J Cogn Psychol 2006; 18: 277-320.

[29] Waldmann MR, Hagmayer Y. Estimating causal strength: the role of structural knowledge and processing effort. Cognition 2001; 821: 27-58.

[30] Shanks DR, Dickinson A. Associative accounts of causality judgment. In: Bower GH, Ed. The psychology of learning and motivation. Advances in research and theory, New York: Academic Press 1987; vol. 21: pp. 229-61.

[31] Wasserman EA, Chatlosh DL, Neunaber DJ. Perception of causal relations in humans: factors affecting judgments of responseoutcome contingencies under free-operant procedures. Learn Motiv 1983; 14: 406-32.

[32] Cheng PW. From covariation to causation: a causal power theory. Psychol Rev 1997; 104: 367-405.

[33] Ahn W, Kalish CW, Medin DL, Gelman SA. The role of covariation versus mechanism information in causal attribution. Cognition 1995; 54: 299-352.

[34] Waldmann MR, Holyoak KJ. Predictive and diagnostic learning within causal models: asymmetries in cue competition. J Exp Psychol Gen 1992; 121: 222-36.

[35] Catena A, Maldonado A, Cándido A. The effect of the frequency of judgment and the type of trials on covariation learning. J Exp Psychol Hum 1998; 24: 481-95.

[36] Maldonado A, Catena A, Cándido A, Garcia I. The belief revision model: Assymmetrical effects of noncontingency on human covariation learning. Learn Behav 1999; 27: 168-80.

[37] Lien Y, Cheng PW. Distinguishing genuine from spurious causes: A coherence hypothesis. Cogn Psychol 2000; 40: 87-137.

[38] Hogarth RM, Einhorn HJ. Order effects in belief updating: The belief-adjustment model. Cogn Psychol 1992; 24: 1-55.

[39] Griffiths TL, Tenenbaum JB. Structure and strength in causal induction. Cogn Psychol 2005; 51: 334-84.

[40] Tenenbaum JB, Griffiths TL, Niyogi S. Intuitive theories as grammars for causal inference. In: Gopnik A, Schulz L, Eds. Causal learning: Psychology philosophy and computation, Oxford: Oxford University Press 2007; pp. 301-22.

[41] Waldmann MR. Competition among causes but not effects in predictive and diagnostic learning. J Exp Psychol Learn 2000; 26: 53-76.

[42] Gigerenzer G, Hoffrage U, Goldstein DG. Fast and frugal heuristics are plausible models of cognition: Reply to Dougherty FrancoWatkins \& Thomas. Psychol Rev 2008; 115: 230-9.

[43] Gigerenzer G, Todd PM, the ABC Research Group. Simple heuristics that make us smart. New York: Oxford University Press 1999.

[44] Todd PM, Gigerenzer G, the ABC Research Group. Ecological rationality: Intelligence in the world. New York: Oxford University Press 2009.

[45] Gigerenzer G, Goldstein DG. Reasoning the fast and frugal way: Models of bounded rationality. Psychol Rev 1996; 103: 650-69.

[46] Gigerenzer G, Goldstein DG. Betting on one good reason: The take the best heuristic. In: Gigerenzer G, Todd PM, the ABC Research Group Eds. Simple heuristics that make us smart. New York: Oxford University Press 1999; pp. 75-95.

[47] Bergert FB, Nosofsky RM. A response-time approach to comparing generalized ration and take-the-best models of decision making. $\mathbf{J}$ Exp Psychol Learn 2007; 33: 107-29.

[48] Bröder A. Assessing the empirical validity of the "take-the-best" heuristic as a model of human probabilistic inference. J Exp Psychol Learn 2000; 26: 1332-46.
[49] Bröder A. Decision making with the "adaptive toolbox": Influence of environmental structure intelligence and working memory load. J Exp Psychol Learn 2003; 29: 611-25.

[50] Bröder A, Gaissmaier W. Sequential processing of cues in memory-based multiattribute decisions. Psychon B Rev 2007; 14: 895-900.

[51] Bröder A, Schiffer S. Bayesian strategy assessment in multiattribute decision making. J Behav Decis Mak 2003; 16: 193-213.

[52] Newell BR, Rakow T, Weston NJ, Shanks DR. Search strategies in decision making: The success of "success". J Behav Decis Mak 2004; 17: 117-37.

[53] Newell BR, Shanks DR. Take the best or look at the rest? Factors influencing "one-reason" decision making. J Exp Psychol Learn 2003; 29: 53-65.

[54] Rieskamp J, Hoffrage U. Inferences under time pressure: How opportunity cost affect strategy selection. Acta Psychol 2008; 127: 258-76.

[55] Garcia-Retamero R, Hoffrage U, Dieckmann A. When one cue is not enough: Combining fast and frugal heuristics with compound cue processing. Q J Exp Psychol 2007; 60: 1197-215.

[56] Rieskamp J, Hoffrage U. When do people use simple heuristics and how can we tell? In: Gigerenzer G, Todd PM, the ABC Research Group Eds. Simple heuristics that make us smart, New York: Oxford University Press 1999; pp. 141-67.

[57] Garcia-Retamero R, Dhami MK. Differences between experts and novices in cue estimations in crime. Psicothema 2009; 21:37681.

[58] Garcia-Retamero R, Dhami MK. Take-the-best in expert-novice decision strategies for residential burglary. Psychon B Rev 2009; 16: 163-9.

[59] Newell BR, Weston NJ, Shanks DR. Empirical tests of a fast-andfrugal heuristic: Not everyone "takes-the-best". Organ Behav Hum Decis 2003; 91: 82-96.

[60] Meder B, Gerstenberg T, Hagmayer Y, Waldmann MR. Observing and intervening: Rational heuristical models of causal decision making. Open J 2010; in press.

[61] Bröder A, Schiffer S. Take the best versus simultaneous feature matching: Probabilistic inferences from memory and effects of representation format. J Exp Psychol Gen 2003; 132: 277-93.

[62] Dieckmann A, Todd PM. Simple ways to construct search orders. Proceedings of the 26th Annual Conference of the Cognitive Science Society, Mahwah NJ: Erlbaum 2004; pp. 309-14.

[63] Todd PM, Dieckmann A. Simple rules for ordering cues in one-reason decision making. In: Todd PM, Gigerenzer G, the ABC Research Group. Ecological rationality: Intelligence in the world, New York: Oxford University Press in press.

[64] Juslin P, Persson M. PROBabilities from EXemplars PROBEX: A "lazy" algorithm for probabilistic inference from generic knowledge. Cogn Sci 2002; 26: 563-607.

[65] Garcia-Retamero R, Hoffrage U, Dieckmann A, Ramos M. Compound cue processing within the fast and frugal heuristic approach in non-linearly separable environments. Learn Motiv 2007; 38: 1634.

[66] Garcia-Retamero R, Wallin A, Dieckmann A. Does causal knowledge help us be faster and more frugal in our decisions? Mem Cogn 2007; 35: 1399-409.

[67] Wallin A, Gärdenfors P. Smart people who make simple heuristics work. Behav Brain Sci 2000; 23: 765.

[68] Boyle P. Cancer cigarette smoking and premature death in Europe: A review including the recommendations of European cancer experts' consensus meeting Helsinki October 1996. Lung Cancer 1997; 17: 1-60.

[69] Pearl J. Causality. New York: Oxford University Press 2000.

[70] Reichenbach H. The direction of time. Berkeley: University of California Press 1956.

[71] Kruschke JK, Johansen MK. A model of probabilistic category learning. J Exp Psychol Learn 1999; 25: 1083-119.

[72] Castellan NJ. Multiple-cue probability learning with irrelevant cues. Organ Behav Hum Perform 1973; 9: 16-29.

[73] Edgell SE, Hennessey JE. Irrelevant information and utilization of event base rates in nonmetric multiple-cue probability learning. Organ Behav Hum Perform 1980; 26: 1-6.

[74] Brehmer B. Note on the relation between single-cue probability learning and multiple-cue probability learning. Organ Behav Hum Perform 1973; 9: 246-52. 
[75] Waldmann MR. Knowledge-based causal induction. In: Shanks DR, Holyoak KJ, Medin DL, Eds. Psychol Learn Motiv. San Diego CA: Academic Press 1996; 34: 47-88.

[76] Waldmann MR, Martignon L. A bayesian network model of causal learning. In: Gernsbacher MA, Derry SJ, Eds. Proceedings of the 20th annual conference of the cognitive science society, Mahwah NJ: Erlbaum 1998; pp. 1102-7.

[77] Lagnado DA, Waldmann MR, Hagmayer Y, Sloman SA. Beyond covariation: Cues to causal structure. In: Gopnik A, Schulz L, Eds. Causal learning: psychology philosophy and computation, Oxford. Oxford University Press 2007; pp. 154-72.

[78] Garcia-Retamero R, Müller SM, Catena A, Maldonado A. The power of causal beliefs and conflicting evidence on causal judgments and decision making. Learn Motiv 2009; 40: 284-97.

[79] Koslowski B. Theory and evidence: the development of scientific reasoning. Cambridge MA: MIT Press 1996.

[80] Koslowski B, Masnick A. The developmental of causal reasoning. In: Goswami U, Ed. Blackwell handbook of childhood cognitive development. Malden MA: Blackwell 2002; pp. 257-81.

[81] Fugelsang JA, Stein CB, Green AE, Dunbar KN. Theory and data interactions in the scientific mind: Evidence from molecular and the cognitive laboratory. Can J Exp Psychol 2004; 58: 8695 .
[82] Martignon L, Hoffrage U. Fast frugal and fit: Simple heuristics for paired comparison. Theor Decis 2002; 52: 29-71.

[83] Catena A, Maldonado A, Perales JC, Cándido A. Interaction between previous beliefs and cue predictive value in covariationbased causal induction. Acta Psychol 2008; 128: 339-49.

[84] Gill MJ. When information does not deter stereotyping: Prescriptive stereotyping can foster bias under conditions that deter descriptive stereotyping. J Exp Soc Psychol 2004; 40: 619-32.

[85] Kawakami K, Dovidio JF, Moll J, Hermsen S, Russin A. Just say no to stereotyping: effects of training in the negation of stereotypic associations on stereotypic activation. Pers Soc Psychol 2000; 78: 871-88.

[86] Maloney JC. Curiosity versus disbelief in advertising. J Adver Res 2000; 40: 7-13

[87] Pratkanis A, Aronson E. Age of propaganda: the everyday use and abuse of persuasion. New York: W H Freeman 2001.

[88] Chapman J. The power of propaganda. J Contemp Hist 2000; 35: 679-88.

[89] Spirtes P, Glymour C, Scheines R. Causation prediction and search. Springer lecture notes in statistics. New York: Springer-Verlag 1993.

[90] Spirtes P, Glymour C, Scheines R. Causation prediction and search. $2^{\text {nd }}$ ed Cambridge. MA: MIT Press 2000..

(C) Garcia-Retamero et al.; Licensee Bentham Open .

This is an open access article licensed under the terms of the Creative Commons Attribution Non-Commercial License (http://creativecommons.org/licenses/by-nc/3.0/) which permits unrestricted, non-commercial use, distribution and reproduction in any medium, provided the work is properly cited. 